\title{
A CLINICO-PATHOLOGICAL STUDY OF LIVER ABSCESS
}

\author{
Sriramoju Sreedhar 1 , Akula Nynasindhu², Sahaja ${ }^{3}$
}

${ }^{1}$ Senior Resident, Department of General Surgery, Mahatma Gandhi Memorial Hospital, Warangal, Telangana.

${ }^{2}$ Senior Resident, Department of General Surgery, Gandhi Medical College, Secunderabad, Telangana.

3Postgraduate Student, Department of General Surgery, Mahatma Gandhi Memorial Hospital, Warangal, Telangana.

\section{BACKGROUND}

ABSTRACT

Liver abscess is a common condition in India. India has 2 nd highest incidence of liver abscess in the world. Liver abscesses are caused by bacterial, parasitic or fungal infection. Pyogenic abscesses account for three-quarters of hepatic abscesses in developed countries, while amoebic liver abscess cause two-thirds of liver abscess in developing countries. Amoebiasis is presently the third most common cause of death from parasitic disease. Primary prevention by improving sanitation, health education, early diagno sis and prompt treatment may result in lowering mortality/ morbidity associated with the disease.

Aims and Objectives- To assess the clinico-pathological and management outcomes in liver abscess patients admitted to General Surgery Department of MGM Hospital between September 2015 and October 2017.

\section{MATERIALS AND METHODS}

The present study was conducted in Mahatma Gandhi Memorial Hospital, Kakatiya Medical College, Warangal, between September 2015 and October 2017. It is a prospective observational study. Detailed history was taken according to the proforma. Patient was followed up conservatively and if complicated then interventional management was done. Patients will be followed up for a minimum period of 6 months.

\section{RESULTS}

Liver abscesses occurred most commonly between 30 - 60 years. Pain abdomen was the most common symptom present in all 100 cases. Alcohol consumption was the single most important aetiological factor for causation of liver abscesses. Raised WBC count, Alkaline phosphatase level, Diabetes, Hypoalbuminaemia and Prolonged prothrombin time were considered as the predictive factors of complicated (ruptured) liver abscess in this study. Amoebic serology when performed using ELISA was positive in significant number of cases making it a strong marker in diagnosing Amoebic liver abscess and also positive in 27 cases of pyogenic liver abscess suggesting significant incidence of mixed infection. The incidence of positive anti-HIV serology was only $3.0 \%$ in this study.

\section{CONCLUSION}

Liver abscess is a very common condition in India with male preponderance. Alcohol is the most common risk factor. Pain abdomen was the most common symptom. Alkaline phosphatase was the most consistent investigation. Most cases had right lobe involvement. Enterococcus was the most common organism. Multiple small abscesses and solitary abscess of volume $<200 \mathrm{cc}$ can be managed successfully on conservative antimicrobial therapy alone, but recurrence rate was high. Ultrasound-Guided Percutaneous Aspiration and Pigtail Catheter drainage procedure is a safe and effective method of liver abscess management. Laparotomy and Drainage or Laparoscopy drainage remains the standard of care in ruptured liver abscess into the peritoneal cavity. Peritoneal rupture was the most common complication associated with Liver abscess.

\section{KEYWORDS}

Amoebic Liver Abscess, Pyogenic Liver Abscess, Pigtail Drainage.

HOW TO CITE THIS ARTICLE: Sreedhar S, Nynasindhu A, Sahaja. A clinico-pathological study of liver abscess. J. Evolution Med. Dent. Sci. 2018;7(11):1376-1379, DOI: 10.14260/jemds/2018/313

\section{BACKGROUND}

Liver abscess is a common condition in India with second highest incidence in the world. Liver abscesses are caused by bacterial, parasitic or fungal infection.

Pyogenic abscesses account for three-quarters of hepatic abscesses in developed countries, ${ }^{1}$ while amoebic liver abscess cause two-thirds of liver abscess in developing countries. $^{2}$

'Financial or Other Competing Interest': None.

Submission 17-01-2018, Peer Review 24-02-2018,

Acceptance 01-03-2018, Published 12-03-2018.

Corresponding Author:

Sriramoju Sreedhar,

\#3-1-72, Kavitha Clinic,

Ganesh Street, Jangaon, Telangana.

E-mail: drsridhar2506@gmail.com

DOI: $10.14260 /$ jemds $/ 2018 / 313$

\section{(c) $(1) \risingdotseq$}

The World Health Organisation reported that Entamoeba histolytica causes approximately 50 million cases and 100,000 deaths annually. 3 The vast majority of these infections are acquired in the developing world. In a country like India where majority of population lives below poverty line, basic sanitary facilities are lacking. This coupled with overcrowding and urban slums and also outdoor unhygienic eating habits sets the stage for communicable diseases like amoebiasis.

Locally made alcoholic drinks like neera, arrack may be the routes of faeco-oral transmission of amoebic cysts.

In the wake of HIV epidemic in our country, this study also tries to investigate the relation between liver abscess and immunocompromised state of AIDS. Primary prevention by improving sanitation, health education, early diagnosis 
and prompt treatment may result in lowering mortality/ morbidity associated with the disease.

\section{Aims and Objectives}

Assess the clinico-pathological and management outcomes in liver abscess patients admitted to General Surgery Department of MGM Hospital between September 2015 and October 2017.

The objective is to study 100 cases of liver abscess and to determine the demographic profile, aetiology, spectrum of clinical presentations and evaluate laboratory investigations profile; to study the bacteriological characteristics including its antibiotic sensitivity; to study the influence of alcohol, diabetes and immunocompromised diseases (esp. HIV) leading to increased incidence of Liver abscess and to evaluate efficacy, recurrence rate, complications, morbidity and mortality, duration of hospital stay associated with management strategies.

\section{MATERIALS AND METHODS}

The present study was conducted in Mahatma Gandhi Memorial Hospital, Kakatiya Medical College, Warangal, between September 2015 and October 2017. It is a descriptive observational study, which included 100 patients who were diagnosed with liver abscess clinically and sonologically and managed with regular follow-up to the OPD.

The patient's data was collected from the OPD, Inpatient wards and a detailed history was noted according to the proforma. Complete haemogram, LFT, Prothrombin time and Serology for amoebic antigen was sent immediately on presentation. Preliminary Ultrasound of Abdomen and Pelvis was done on the same day of presentation. Patient was put on conservative line of management. If abscess was more than $200 \mathrm{cc}$, then percutaneous needle aspiration was done.

Patient was followed up daily clinically and LFT and USG abdomen was repeated on the $3^{\text {rd }}$ day if patient is symptomatically not relieved. Repeat Ultrasound/ CT/ MRI abdomen and pelvis will be done immediately if patient's condition does not improve/ worsen or after 3 - 4 days as a routine.

If the patient develops any of the complications like ruptured liver abscess into any of the serosal cavities, patient was immediately taken up for surgery (Laparotomy drainage or Laparoscopic drainage). Pus was sent for Gram's stain and culture and sensitivity. Anaerobic cultures were not done as the facility was not available in our hospital. Patient was informed about any surgical procedure and consent taken.

\section{Patients will be followed up for a Minimum Period of 6 Months-}

1. Monthly for first 3 months.

2. Then once after 6 months for recurrent attacks or development of complications and to monitor the efficacy of the treatment given.

Cure was defined as improvement clinically with subsidence of fever, and local signs, symptoms, decrease in WBC count and follow-up ultrasonography showed reduction in size $<3 \mathrm{~cm}$ in diameter and no evidence of relapses.

\section{Statistical Methods}

Descriptive statistical analysis has been carried out in the present study. Results on continuous measurements are presented on Mean ? SD (Min-Max) and results on categorical measurements are presented in Number (\%). Significance is assessed at 5\% level of significance. Chisquare/ Fisher Exact tests have been used to find the significance of study parameters on categorical scale between two or more groups. 95\% Confidence Interval has been computed to find the significant features. Confidence Interval with lower limit more than $50 \%$ is associated with statistical significance.

\section{RESULTS}

In the present study, the mean age was 46 years. The highest incidence was noted in the age group of 31 - 40 years $(25.0 \%)$ and 51 - 60 years (25\%). 89\% of patients were male and $11 \%$ were female. $60 \%$ of patients presented with acute symptoms, whereas $37 \%$ presented with subacute symptoms. Abdominal pain was present in all cases. Fever was the most consistent symptom occurring in $94 \%$. Diarrhoea occurring in $16 \%$, jaundice was present in $24 \%$ of patients. $30 \%$ had respiratory symptoms. Tender, smooth, soft-to-firm consistency hepatomegaly was seen in $50 \%$ of cases. $4 \%$ presented with features of shock. $20 \%$ of cases had an intraperitoneal rupture. $3 \%$ presented with cholangitis and pleural rupture each. $84 \%$ of the patients are alcoholic.

$72 \%$ of the patients had WBC $>11,000$. Anaemia $(\mathrm{Hb}<10$ $\mathrm{gm} / \mathrm{dL}$ ) were found in $23.0 \%$ of the cases. $29 \%$ were found to be diabetic with RBS $>200 \mathrm{mg} / \mathrm{dL}$. Raised urea (> $45 \mathrm{mg} / \mathrm{dL}$ ) was found in $21 \%$ of the cases. Serum creatinine $(>1.4 \mathrm{~g} / \mathrm{dL})$ was seen in $16 \%$ cases. Liver function tests were done in all 100 patients included in this study.

Hyperbilirubinaemia with serum bilirubin $>2.4 \mathrm{gm} / \mathrm{dL}$ was found in 23/100 (23\%) of the cases in this study. Alkaline phosphatase was found to be raised in $83 / 100$ $(83.0 \%)$ of cases. Hypoalbuminaemia $(<3 \mathrm{gm} / \mathrm{dL})$ was observed in 84/100 (84\%). Increased prothrombin time $>20$ sec was seen in 29/100 (29\%) of cases. Increased SGOT and SGPT was seen in $52 \%$ and $45 \%$ of the cases in this study.

Enterococcus was the most common organism cultured in our study accounting for $34 \%$. Out of 30 culture positive cases of Pyogenic liver abscess, 27 cases came as amoebic serology positive. $3 \%$ of patients were found to be HIV positive. Abnormal chest x-ray findings was present in $61 \%$ cases. Solitary abscess was observed in 78.0\%. Isolated right lobe abscess were seen in $74.0 \%$. Multiple abscesses were seen in $22 \%$ of cases. The following line of management was done.

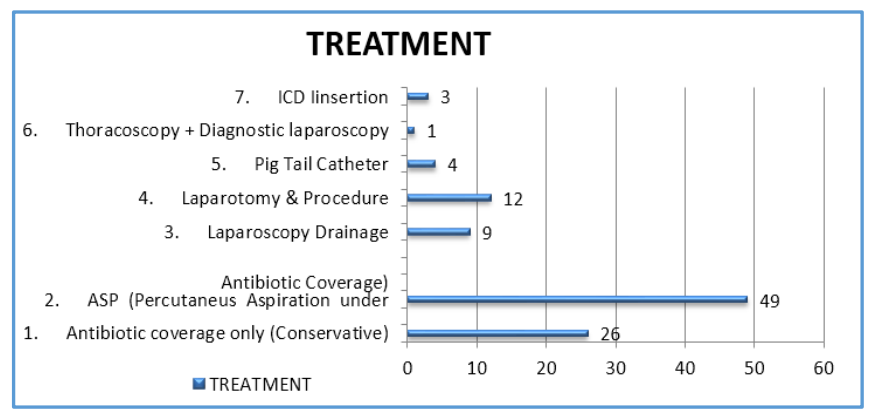


Alcoholism ( $>10$ yrs.), Alkaline Phosphatase $(>300$ $\mathrm{IU} / \mathrm{mL}$ ), Albumin $(<2.0 \mathrm{mg} / \mathrm{dL})$, Prothrombin time $(>20$ seconds), Total leucocyte count ( $>20,000 \mathrm{cc} / \mathrm{mm})$, Pleural effusion and Hospital stay ( $>3$ weeks) were found to have strong correlation with complicated liver abscess with statistically significant as $\mathrm{P}$ value $<0.001$, which shows they are good predictive marker of complications associated with liver abscess (ruptured liver abscess, mean duration of follow-up was 12 months with recurrence seen in $8 \%$ of cases.

\section{DISCUSSION}

In 1938, Ochsner's classic review heralded surgical drainage as the definitive therapy; however, despite the more aggressive approach to treatment, the mortality rate remained at $60 \%-80 \%$. 1

India being a tropical country and home to 400 million people harbouring E. histolytica, the causative organism of amoebic liver abscess, it assumes immense importance for thorough understanding of the same.

The changing scenario in incidence, diagnostic methods, treatment and complications associated with liver abscess due to increasing percentage of alcoholics and immunecompromised population, the current serious problem in our country has inspired me in doing an in depth study regarding Liver Abscesses which assumes more importance in our country where rural population constitutes approximately $70 \%$, and therefore it mandates appropriate and realistic guidelines to be drawn up for early diagnosis and change in management strategies in order to reduce the morbidity and mortality associated with it.

\section{Age Incidence}

\begin{tabular}{|c|c|}
\hline Studies & Mean Age in Years \\
\hline Shyam Mathur ${ }^{4}$ & 32.5 \\
\hline Khee Siang Chang Sing Ming $^{5}$ & 47.6 \\
\hline Antonia Grorgia & \\
\hline Present Study & 45.3 \\
\hline
\end{tabular}

\section{Duration of Onset}

\begin{tabular}{|c|c|c|}
\hline Onset of Disease & $\begin{array}{c}\text { Sumit Kapadia, } \\
\text { Dipesh Dattaroy et } \\
\mathrm{al}^{7}\end{array}$ & Present Study \\
\hline$<7$ days & - & $\begin{array}{c}\text { Most patients } \\
(60 \%)\end{array}$ \\
\hline $\begin{array}{c}7 \text { days }-2 \text { months } \\
\text { (subacute) }\end{array}$ & Most patients & $37 \%$ \\
\hline$>2$ months & - & $3 \%$ \\
\hline
\end{tabular}

RUQ Tenderness (100.0\%), Fever (94.0\%) and Hepatomegaly (50.0\%) was common presentation in our series and was comparable to the studies listed below, but Jaundice $(24.0 \%)$ was more common clinical presentation compared to study done by Hyo Min Yoo et $\mathrm{al}^{4}$ (7.0\%). In our study, cavity size was $>5 \mathrm{~cm}$ in $74 \%$ of patients as compared to $55 \%$ in Hyo Min Yoo et al.

Size of cavity has direct relation to the level of ALP as studied by Anil Kumar $S$ et al. ${ }^{5}$

$17.56 \%$ showed Klebsiella pneumoniae as culture, whereas in Hiroshi Okana et al $62 \%$ were positive for
Klebsiella pneumoniae, $92.94 \%$ were positive for amoebic serology comparable to Iranian J Public Health 2003.7 Chest $\mathrm{x}$-ray findings were comparable to those with D. Lynche, William et $\mathrm{al}^{8}$ isolated left lobe involvement was $04 \%$ in our study as compared to other study Hyo Min Yoo et al (20.0\%) and Chaturbhul Lal Rajak et al 12.0\%. ${ }^{9}$

In majority of cases, percutaneous aspiration was the main form of treatment. All patients were started on antibiotics, which were continued for 10 - 14 days depending on improvement. Majority of patients responded excellently to percutaneous aspiration and antimicrobials. While patients who had smaller abscesses or multiple small abscesses were successfully managed with antimicrobial therapy alone, but relapse rates were high. Hospital stay ( $>3$ weeks) were found to have strong correlation with complicated liver abscess with statistically significant as $p$ value $<0.001$. The factors associated with complicated liver abscess were comparable with other studies. In our study, Cryptogenic was the most common aetiology of liver abscess (Amoebic and Pyogenic).

Gerald Y Minuk, MD et $\mathrm{al}^{10}$ in their study suggested that patients who develop cryptogenic abscesses of the liver have an underlying reticuloendothelial cell defect that may predispose them to liver abscess formation.

\section{CONCLUSION}

Liver abscess is a very common condition in India. India has 2nd highest incidence of liver abscess in the world. Liver abscesses occurred most commonly between 30 - 60 years. Most of the cases had an acute presentation. Males were affected more than females. Pain abdomen was the most common symptom present in all 100 cases. Fever being the most consistently occurring symptom. Alcohol consumption was the single most important aetiological factor for causation of liver abscesses.

Alkaline phosphatase is the most consistently elevated among all Liver Function Tests. Raised WBC count, Alkaline phosphatase level, Diabetes, Hypoalbuminaemia and Prolonged Prothrombin time were considered as the predictive factors of complicated (Ruptured) liver abscess in this study. Diabetes mellitus was more frequently associated condition in cases of liver abscess and especially pyogenic liver abscess cases.

Liver abscess usually presents as a solitary abscess, most commonly in the right lobe of liver. Amoebic serology when performed using ELISA was positive in significant number of cases making it a strong marker in diagnosing Amoebic liver abscess and also positive in 27 cases of pyogenic liver abscess suggesting significant incidence of mixed infection. The incidence of positive anti-HIV serology was only 3.0\% in this study. Enterococcus was the most common organism isolated in pyogenic liver abscess.

All cases of liver abscesses do not require invasive management. Multiple small abscesses and solitary abscess of volume $<200$ cc can be managed successfully on conservative antimicrobial therapy alone, but recurrence rate was high. Ultrasound-Guided Percutaneous Aspiration and Pigtail Catheter drainage procedure is a safe and effective method of liver abscess management. Laparotomy and Drainage or Laparoscopy drainage remains the standard of care in ruptured liver abscess into the peritoneal cavity in this study, as we had no recurrence and mortality associated 
with it. Cryptogenic was the most common aetiology in Amoebic liver abscess as well as in Pyogenic liver abscess. Peritoneal rupture was the most common complication associated with Liver abscess. There was no mortality rate in this study.

\section{REFERENCES}

[1] Ochsner A, DeBakey M, Murray S. Pyogenic abscess of the liver II. An analysis of forty-seven cases with review of the literature. Am J Surg 1938;40(1):292319.

[2] Kapoor OP. Amoebic liver abscess. 1 $1^{\text {st }}$ edn. Bombay: SS Publishers 1999.

[3] Davis A, Pawloski ZS. Amoeblasis and its control: A WHO meeting. Bulletin of World Health Organ 1985;63(3):417-26.

[4] Yoo HM, Kim WH, Shin SK, et al. The changing patterns of liver abscess during the past 20 years - a study of 482 cases. Yonsei Medical Journal 1993;34(4):340-51.
[5] Kumar AS, Mishra A, Malhotra N, et al. Hyperbilirubinemia in patients with amoebic liver abscess: a study of 75 cases. J Gastroint \& Dig Syst 2013;3:38.

[6] Okano H, Shiraki K, Inoue H, et al. Clinico pathological analysis of liver abscess in Japan. International Journal of Molecular Medicine 2002;10(5):627-30.

[7] Arianpour N, Mohapatra TM. Study of antiamoebic antibody in amoebiasis using ELISA and RID Techniques. Iranian J Publ Health 2003;32(4):13-8.

[8] Lynche KD, Jensen WA, Kirsch CM, et al. Pleuro pulmonary manifestations of hepatic amebiasis. West J Med 1990;153(3):275-8.

[9] Rajak CL, Gupta S, Jain S, et al. Percutaneous treatment of liver abscess: needle aspiration versus catheter drainage. American Journal of Roentgenology 1998;170(4):1035-9.

[10] Minuk GY, Nicole LE, Sherman T. Cryptogenic abscess of the liver evidence of underlying reticuloendothelial cell dysfunction. Arch Surg 1987;122(8):906-8. 